\title{
Transcriptome characterisation of Pinus tabuliformis and evolution of genes in the Pinus phylogeny
}

\author{
Shi-Hui Niu' ${ }^{1}$, Zhe-Xin Li ${ }^{1}$, Hu-Wei Yuan ${ }^{1}$, Xiao-Yang Chen ${ }^{1,2}$, Yue Li ${ }^{1}$ and Wei Li ${ }^{1 *}$
}

\begin{abstract}
Background: The Chinese pine (Pinus tabuliformis) is an indigenous conifer species in northern China but is relatively underdeveloped as a genomic resource; thus, limiting gene discovery and breeding. Large-scale transcriptome data were obtained using a next-generation sequencing platform to compensate for the lack of P. tabuliformis genomic information.
\end{abstract}

Results: The increasing amount of transcriptome data on Pinus provides an excellent resource for multi-gene phylogenetic analysis and studies on how conserved genes and functions are maintained in the face of species divergence. The first $P$. tabuliformis transcriptome from a normalised cDNA library of multiple tissues and individuals was sequenced in a full 454 GS-FLX run, producing 911,302 sequencing reads. The high quality overlapping expressed sequence tags (ESTs) were assembled into 46,584 putative transcripts, and more than 700 SSRs and 92,000 SNPs/InDels were characterised. Comparative analysis of the transcriptome of six conifer species yielded 191 orthologues, from which we inferred a phylogenetic tree, evolutionary patterns and calculated rates of gene diversion. We also identified 938 fast evolving sequences that may be useful for identifying genes that perhaps evolved in response to positive selection and might be responsible for speciation in the Pinus lineage.

Conclusions: A large collection of high-quality ESTs was obtained, de novo assembled and characterised, which represents a dramatic expansion of the current transcript catalogues of $P$. tabuliformis and which will gradually be applied in breeding programs of $P$. tabuliformis. Furthermore, these data will facilitate future studies of the comparative genomics of $P$. tabuliformis and other related species.

Keywords: Pinus tabuliformis Carr, 454 pyrosequencing, SNPs, SSRs, Pinus phylogeny, Comparative transcriptomics

\section{Background}

Conifers are widely distributed globally as the largest and most diverse group of gymnosperms [1] that evolved independently from angiosperms $>300$ million years ago [2]. Modern conifers are divided into eight families including 68 genera and 630 species, which form an integral part of the economy in many parts of the world [3]. Chinese pine (Pinus tabuliformis Carr.) is a widespread indigenous conifer species and an economically and ecologically important hard pine in northern China $[4,5]$.

\footnotetext{
* Correspondence: bjfuliwei@bjfu.edu.cn

${ }^{1}$ National Engineering Laboratory for Forest Tree Breeding, Key Laboratory for Genetics and Breeding of Forest Trees and Ornamental Plants of the Ministry of Education, College of Biological Science and Technology, Beijing Forestry University, Beijing 100083, People's Republic of China

Full list of author information is available at the end of the article
}

Because of its irreplaceable economic development and environmental protection status, a genetic improvement program for $P$. tabuliformis was initiated in the 1970s, and considerable progress has been made in many basic physiological aspects [4]. The study of natural genetic variation in $P$. tabuliformis has traditionally been investigated using a common garden approach, whereas the pace of development of genomic resources has been slow, as only $288 P$. tabuliformis entries are included in the NCBI database. Information regarding the genetic control of many important traits and fine-scale genetic variations is extremely limited, and more is needed given the renewed emphasis to accelerate the pace of $P$. tabuliformis breeding and shorten the breeding cycle.

Despite the economic and ecological importance of the genus Pinus, the progress of entire genome sequencing 
and associated marker development has been limited [6,7]. Huge genomes with highly heterozygous and large amounts of repetitive DNA elements are the major obstacles towards sequencing the genomes of all Pinus spp. $[8,9]$. The genome sizes of conifers are larger than those of most other plant species. The genome in all extant members of the genus Pinus is 18,000-40,000 Mbp [10]. In contrast, several representative genera of angiosperm trees have genome sizes of 540-2,000 Mb [1]. Therefore, researchers have focused on the transcribed part of the genome using dedicated technologies [6,7]. Transcriptome analysis and construction of large-scale expressed sequence tag (EST) collections in pines are a promising means of providing genomic resources $[2,9,11]$, as this technique produces expressed sequence portions of chromosomes at a fraction of the cost of sequencing the complete genome [12]. It also facilitates the analysis of the transcribed part of the genome, which is not easy to predict from the entire genome [13]. Next-generation sequencing is a viable and favourable alternative to Sanger sequencing and provides researchers with a relatively rapid and affordable option for developing genomic resources in non-model organisms [14-16]. The Roche 454 massively parallel pyrosequencing platform, GS FLX Titanium, can generate one million reads with an average read length of 400 bases at $99.5 \%$ accuracy per run $[17,18]$.

In addition to the discovery of new genes and investigations of gene expression, thousands of simple sequence repeats (SSRs), single nucleotide polymorphisms (SNPs) and insertions and deletions (Indels) have been detected in transcriptome data $[6,19]$. It is possible to use these genome-wide and abundant markers to develop very dense genetic maps that can be applied to conduct marker-assisted selection breeding programs [20].

Moreover, the increasing availability of transcriptome data represents an excellent resource for comparative genomic analysis. Although there has been much work on the chloroplast DNA sequences (cpDNA) and mitochondria DNA sequences (mtDNA), based on phylogenetic analysis of Pinus [21-23], less emphasis has been placed on multi-gene phylogenetic analysis and on determination of how conserved genes and functions are maintained despite species divergence.

In the current study, we used the Roche 454 GS-FLX Titanium pyrosequencing platform to obtain a comprehensive transcriptome of $P$. tabuliformis from normalised cDNA libraries of adult trees (xylem, phloem, vascular cambium, needles, cones and strobili). As a result, thousands of molecular markers were characterised. Evolutionary studies based on these data and other shared transcriptome data of five pine species and one spruce species were conducted. These data provide compelling new insights into the transcriptome of P. tabuliformis and evolution of genes in the Pinus phylogeny.

\section{Results}

Transcriptome sequencing and de novo assembly

Prior to sequencing, the cDNA samples obtained from multiple tissues and individuals were normalised to increase the sequencing efficiency of rare transcripts. Subsequently, 911,302 raw reads with an average length of 382 bp were generated from a full 454 GS-FLX run. After a trimming process removed adaptors, primer sequences, poly-A tails as well as short, long and low quality sequences, 822,891 (84.7\%) high-quality reads were obtained with an average length of 358 bp covering a total of 21,076,176 bases (Table 1, Figure 1a). Cleaned and qualified reads were de novo assembled using CAP3 and Newbler. This process produced a set of 31,623 isotigs and 17,853 remaining as singletons. More than half of the total assembly length of isotigs was $>700 \mathrm{bp}$ $(\mathrm{N} 50=744)$ (Table 1, Figure 1b).

The unigene coverage distribution revealed that most unigenes had a read-depth coverage $<20$-fold (Figure 1c, d). The steep decline in read-depth coverage suggests that cDNA normalisation was effective, which is typical for a normalised library [24]. Isotig lengths were related to the number of sequences assembled into each isotig. The average unigene length exhibited a gradual increase with increasing read depth (Figure 1c, d).

Table 1 Sequencing, assembly and data analysis

\begin{tabular}{llll}
\hline \multicolumn{2}{c}{ Raw results (after trimming) } & & \multicolumn{2}{c}{ Assembly results } \\
\hline Total number of reads & 822891 & Total number of isotigs & 31623 \\
\hline Total read length (bp) & 295125234 & Total isotigs length (bp) & 21076176 \\
\hline Minimum read length (bp) & 50 & Isotig N50 (bp) & 744 \\
\hline Median read length (bp) & 384 & Maximum isotig length (bp) & 3537 \\
\hline Maximum read length (bp) & 578 & Mean depth & 28.2 \\
\hline Mean read length (bp) & 358 & Number of singletons & 17853 \\
\hline GC content (\%) & 43.2 & Total number of unigenes & 46584 \\
\hline
\end{tabular}




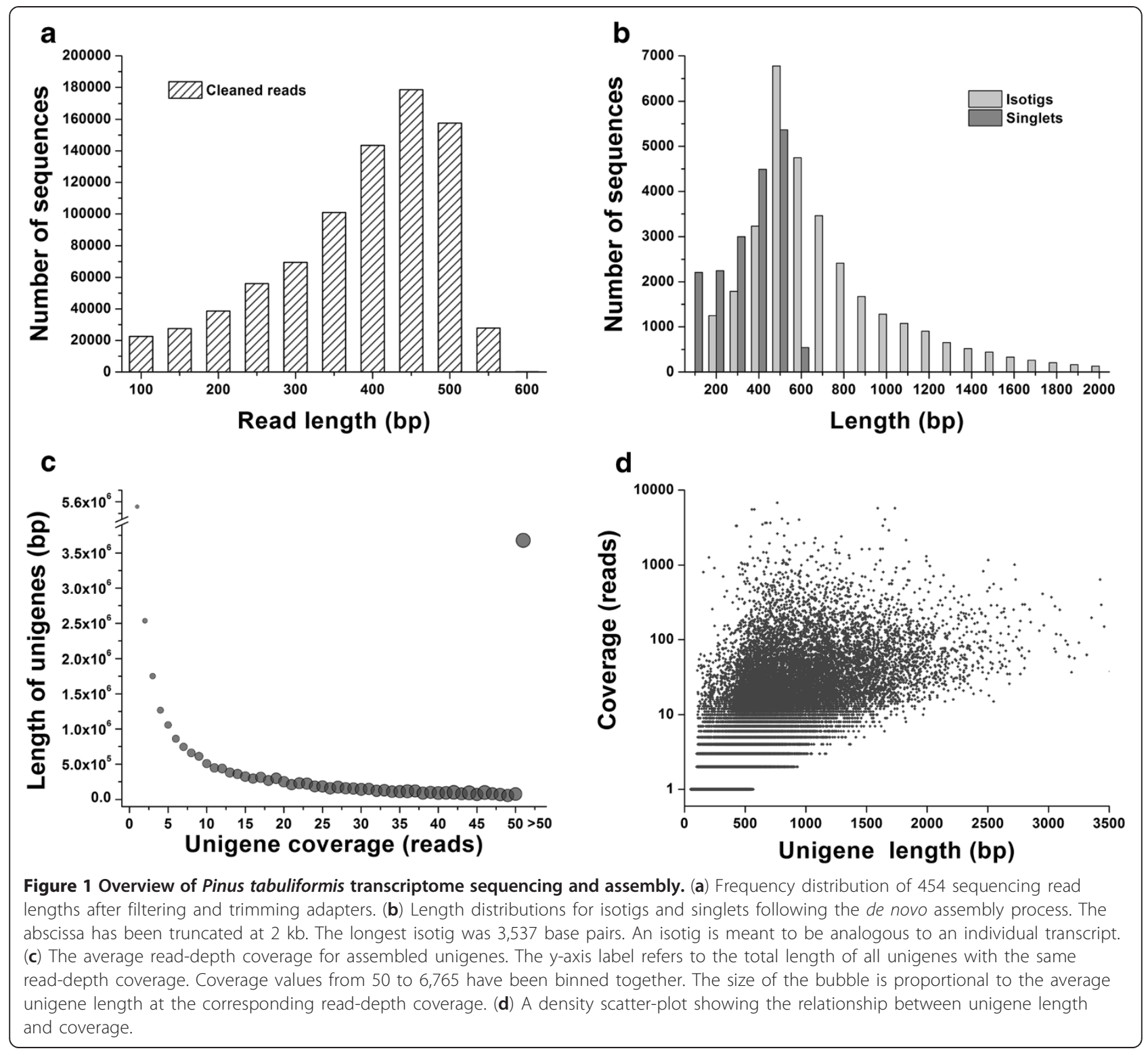

\section{Functional annotation of the transcriptome}

The unigenes were annotated with gene names and Gene Ontology (GO) terms based on sequence comparisons between $P$. tabuliformis transcripts and the NCBI non-redundant protein database. We examined the taxonomic distribution of BLASTx best hits. As a result $99.3 \%(21,041)$ of the unigenes had a best hit to Pinaceae, but $95 \%$ were unknown functional proteins. Of the 3,151 genes with specific functional annotation, 18.9\% were within Pinus and 9.7\% were within Picea (Figure 2). The even distribution of assignments of proteins to more specialised GO terms further indicates that the $P$. tabuliformis 454 sequences represent proteins from a diverse range of functional classes (Figure 3).

\section{Identification of SSRs, SNPs and Indels}

Di- to hexa-nucleotide SSRs with a minimum repeat unit size of five (for tri- to hexa-nucleotide) or six (for dinucleotide) were identified based on the analysis of assembled isotig templates. A total of 724 distinct loci were identified, and the incidences of different repeat types were determined. The tri-nucleotide repeats were most abundant (62.2\%), followed by di-nucleotides (33.7\%), among the various classes of SSRs (Figure 4).

More than 92,000 SNPs/Indels were identified (61,454 SNPs and 31,030 Indels) from the P. tabuliformis ESTs. The number of SNPs/Indels detected per transcript was highly variable; however, approximately $40 \%$ of the transcripts contained only one or two SNPs/Indels (Figure 5a). Among all SNPs, transitions (69.5\%) were more frequent 


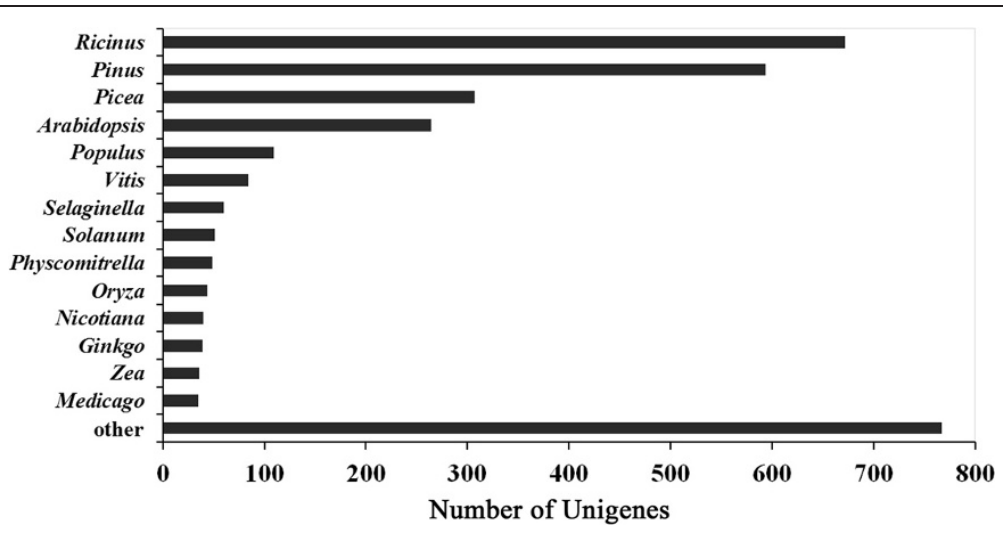

Figure 2 Summary and taxonomic source of BLASTx matches to unigenes. Number of unique best BLASTx matches of unigenes grouped by genus. The best matches of the unigenes to Pinaceae sequences accounted for $28.6 \%$ of the total.

than transversions (30.5\%) (Figure $5 \mathrm{~b})$. A and $\mathrm{T}$ were the most frequent insertion (76.7\%) and deletion (79.5\%) types of InDels (Figure 5c). The distribution of alternate allele frequencies in all contigs containing InDels was less frequent in total transcripts, but the SNPs were distributed evenly (Figure 5d).

\section{Orthologue identification and functional characterisation between six conifer species}

Large-scale transcriptome characterisations have been carried out for Pinus taeda [3], Pinus contorta [25], Pinus sylvestris [26] and Pinus pinaster [2]. The shared transcriptomes of Pinus in the PlantGDB and NCBI databases are valuable sources of information for multigene comparative and phylogenetic analyses [27].

A comparative analysis of the transcriptomes of $P$. tabuliformis, P. contorta, P. pinaster, P. sylvestris, P. taeda and Picea glauca yielded 191 putatively orthologous sets of ESTs (Additional file 1). The orthologues were annotated with GO terms, and 54 orthologues were involved in biological processes, 33 orthologues were involved in cellular components, 54 orthologues were involved in molecular functions and the other 50 orthologues had unknown biological functions (Figure 6).

\section{Phylogenetic and speciation analysis}

Phylogenetic analyses of Pinus species and Picea glauca as an out-group were conducted from 191 clusters of orthologous transcripts, using non-synonymous substitution rates as a distance metric. The results in Figure 7 show good agreement with classical taxonomy. Similar concordance was observed in the cpDNA and mtDNAbased reconstructions of the Pinus phylogeny [21,22].

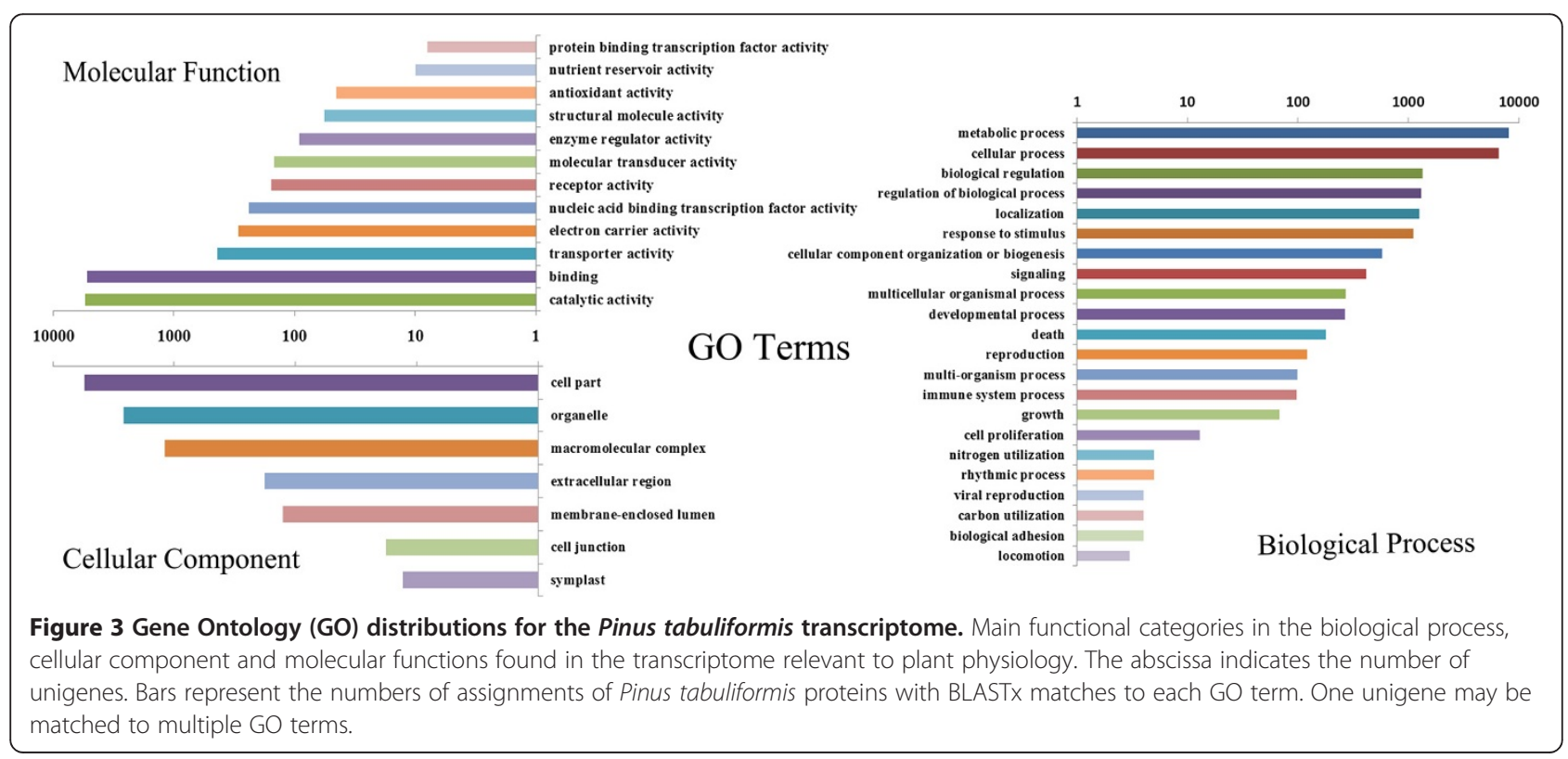




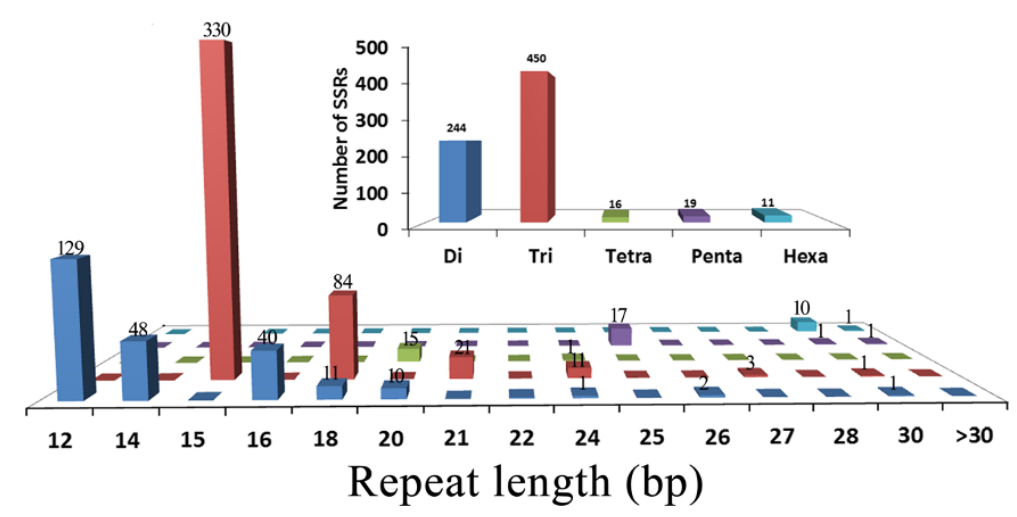

Figure 4 Distribution of simple sequence repeats (SSRs) in Pinus tabuliformis expressed sequence tags (ESTs). Di-, tri-, tetra-, penta- and hexa-nucleotide repeats were analysed and their frequencies plotted as a function of the repeat number. The upper right histogram shows the distribution of the total number of SSRs in different classes.

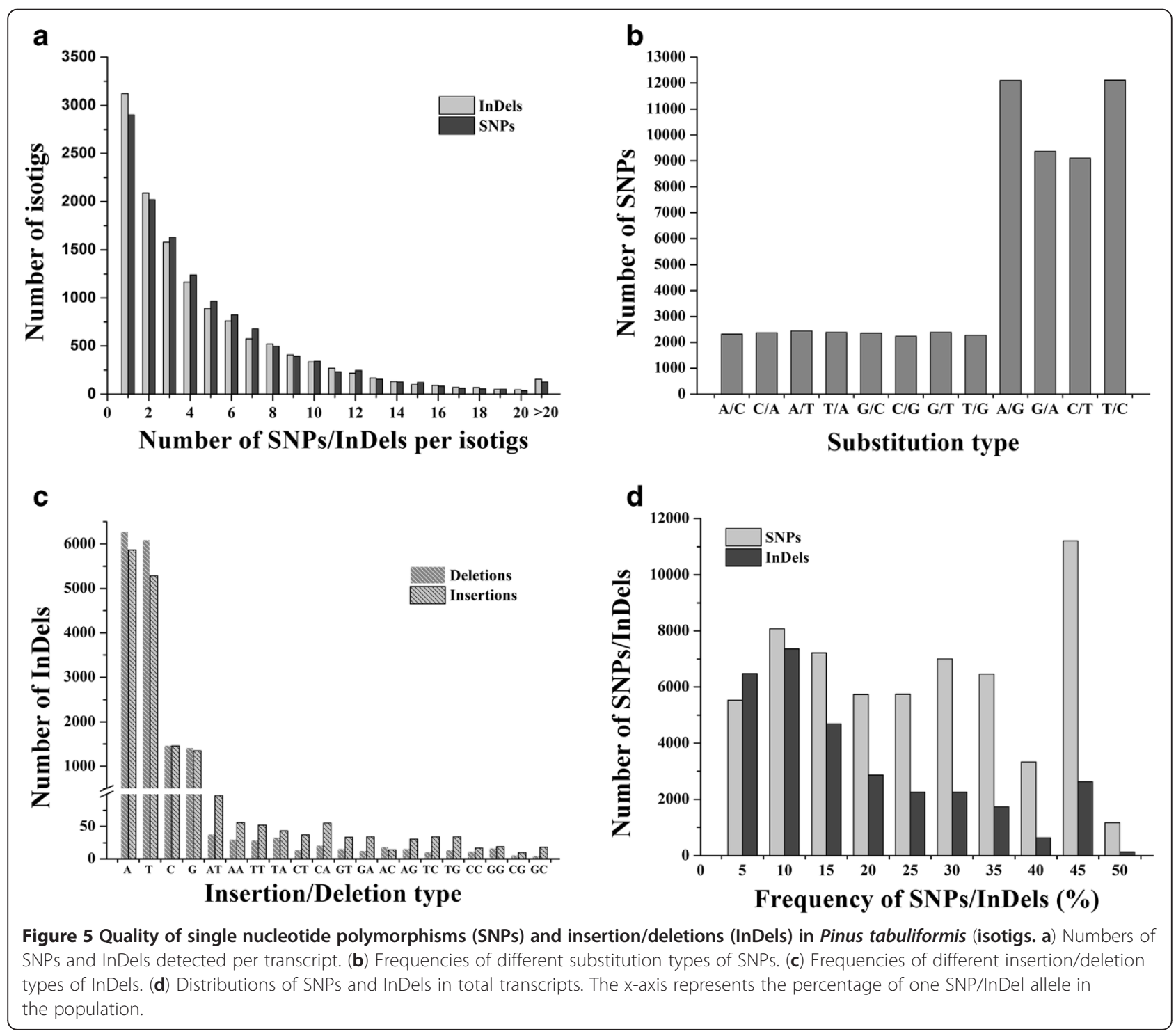




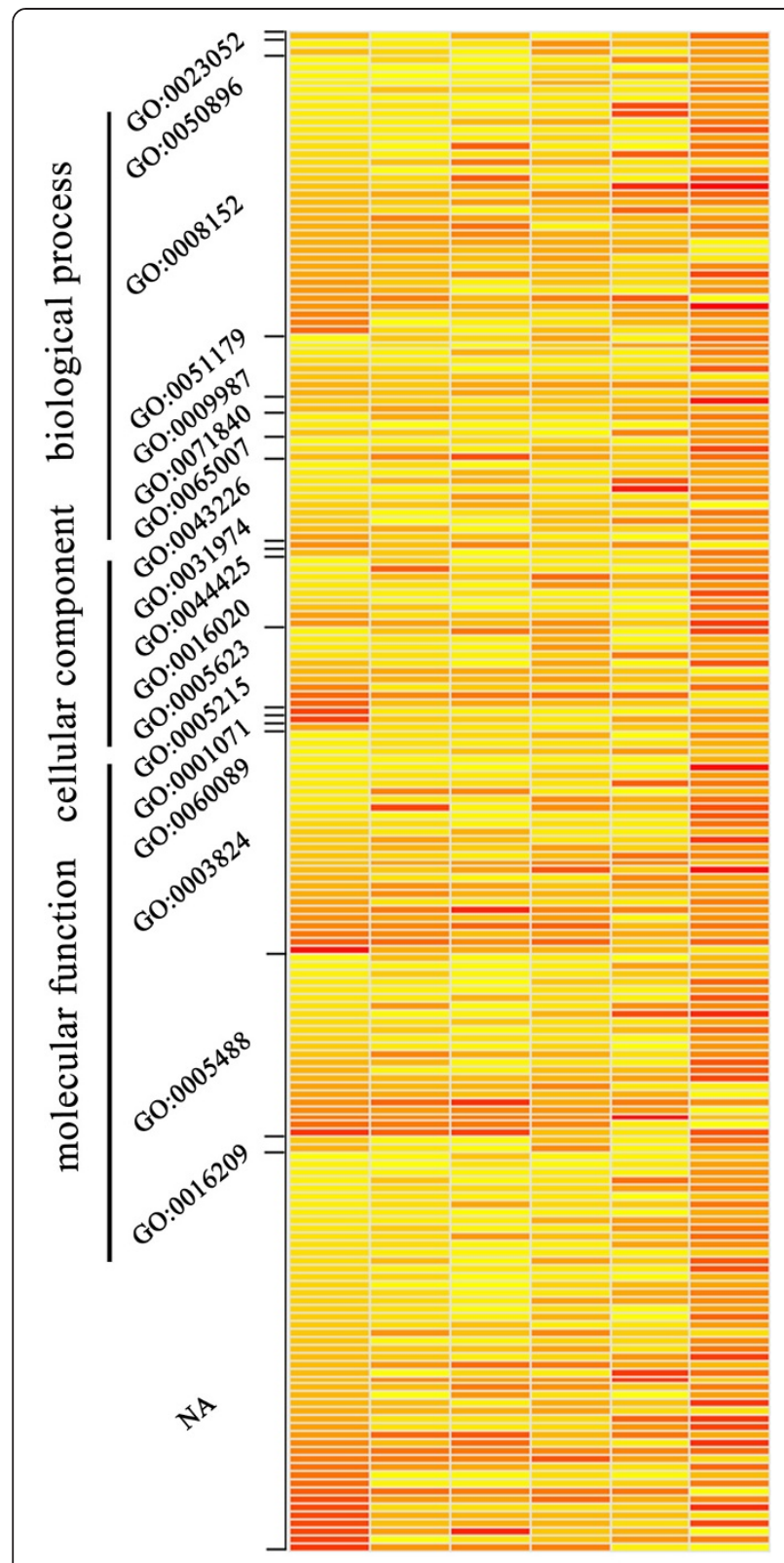

$2 \mathrm{x}$ average

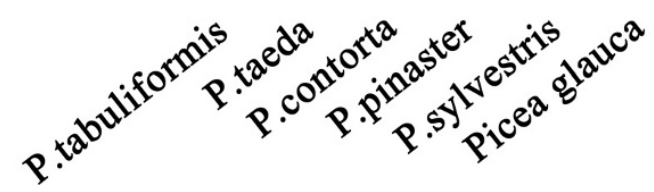

Figure 6 Functional annotation and divergence between homologs of five pine and one spruce species. The heat map is based on the 191 putatively orthologous transcripts of six species. The homologs were annotated with Gene Ontology (GO) terms. Colours indicate similarity from yellow (highly similar) to red (weakly similar). The " $2 \times$ average" is an overall measure of how similar the different species are.
We estimated the level of synonymous substitutions for 191 pairs of orthologues identified among the six species and 6,053 pairs of orthologues identified between $P$. tabuliformis with $P$. taeda as a control to assess the relative age of these species separations. The Ks peaks (Picea glauca $=0.1, P$. taeda and $P$. contort $=0.03, P$. pinaster and $P$. sylvestris $<0.01)$ indicate the speciation time between $P$. tabuliformis and these species (Figure 8). Considering a clock-like synonymous mutation rate of $0.68 \times 10^{-9}$ substitutions/site/year in conifer genes based on pairwise comparisons of 3,723 spruce (Picea sitchensis) and pine (P. taeda) orthologues [28], the speciation between spruce and pine was estimated to have occurred 147 million years ago (mya) and between section Trofoliae and section Pinus $\sim 44$ mya ago.

\section{Evolutionary pattern of Pinus spp. genes}

We estimated evolutionary measures at 6,053 orthologues of $P$. tabuliformis and P. taeda. The number of pairwise synonymous $(K s)$ and non-synonymous $(K a)$ substitutions per site was inferred (Figure 9). The results show that a majority of sequence pairs (85\%) had a $\mathrm{Ka}$ / $K s$ ratio $<1$, suggesting that these evolved under purifying selection without altering the encoded amino acid sequence during the speciation period. We also identified 938 fast evolving sequences with a $K a / K s$ ratio $>1$ and 207 sequences with $\mathrm{Ka} / \mathrm{Ks}$ ratios $>2$ (Additional file 2). These sequences are related to several biological processes, cellular components and molecular functions. Therefore, these ESTs may be useful for identifying genes that may have evolved in response to positive selection and might be responsible for speciation in the Pinus lineage.

\section{Discussion}

A large number of ESTs for pines have been sequenced to date (Table 2). Due to the economic value of wood and pulp products, the initial EST projects on pine focused primarily on the transcriptional regulation of wood formation [29]. Large numbers of ESTs have been sequenced and analysed in pine to discover wood formation and wood quality trait related genes [30-33]. Sequencing novel genes expressed during wood formation represents a powerful approach to understanding wood formation at the molecular level and identifying the mechanisms that control this important differentiation pathway. A total of 260 differentially expressed sequences have been identified across six cDNA libraries from the xylem of P. taeda [34]. A large number of represented gene sequences from xylem-forming tissues of loblolly pine have been compared with the inferred gene sequences of Arabidopsis thaliana [33]. In addition, 42 EST resembled gene products important for drought tolerance have been identified from root tissue libraries [35]. To study similarities between angiosperm and 


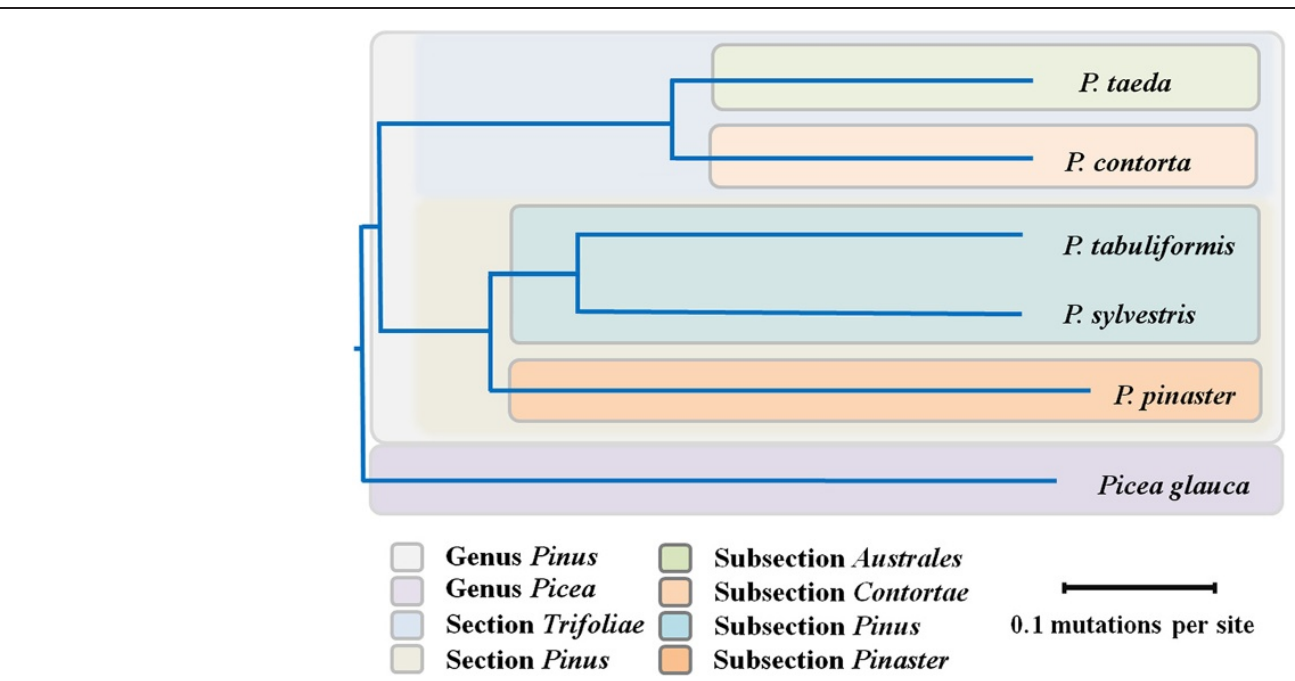

Figure 7 Phylogram of the five pine and one spruce species. Phylogram derived using pairwise non-synonymous substitution rates of orthologous transcripts as a distance metric (not from multiple sequence alignments) and the neighbour-joining method [66]. Branch lengths indicate the non-synonymous substitution rates between different species.

gymnosperm embryo development, 83 embryogenesisrelated genes were identified from embryo cDNA libraries [3]. Most genomic studies of pines have focused on loblolly pine, while additional sequencing efforts are needed to develop genomic resources for other pines.
Despite the fact that a large number of pine ESTs have been obtained from cDNA libraries based on traditional sequencing technology, the methods used were inefficient. Four $P$. taeda cDNA libraries were sequenced and yielded a total of 142,533 ESTs (Table 2); however, only

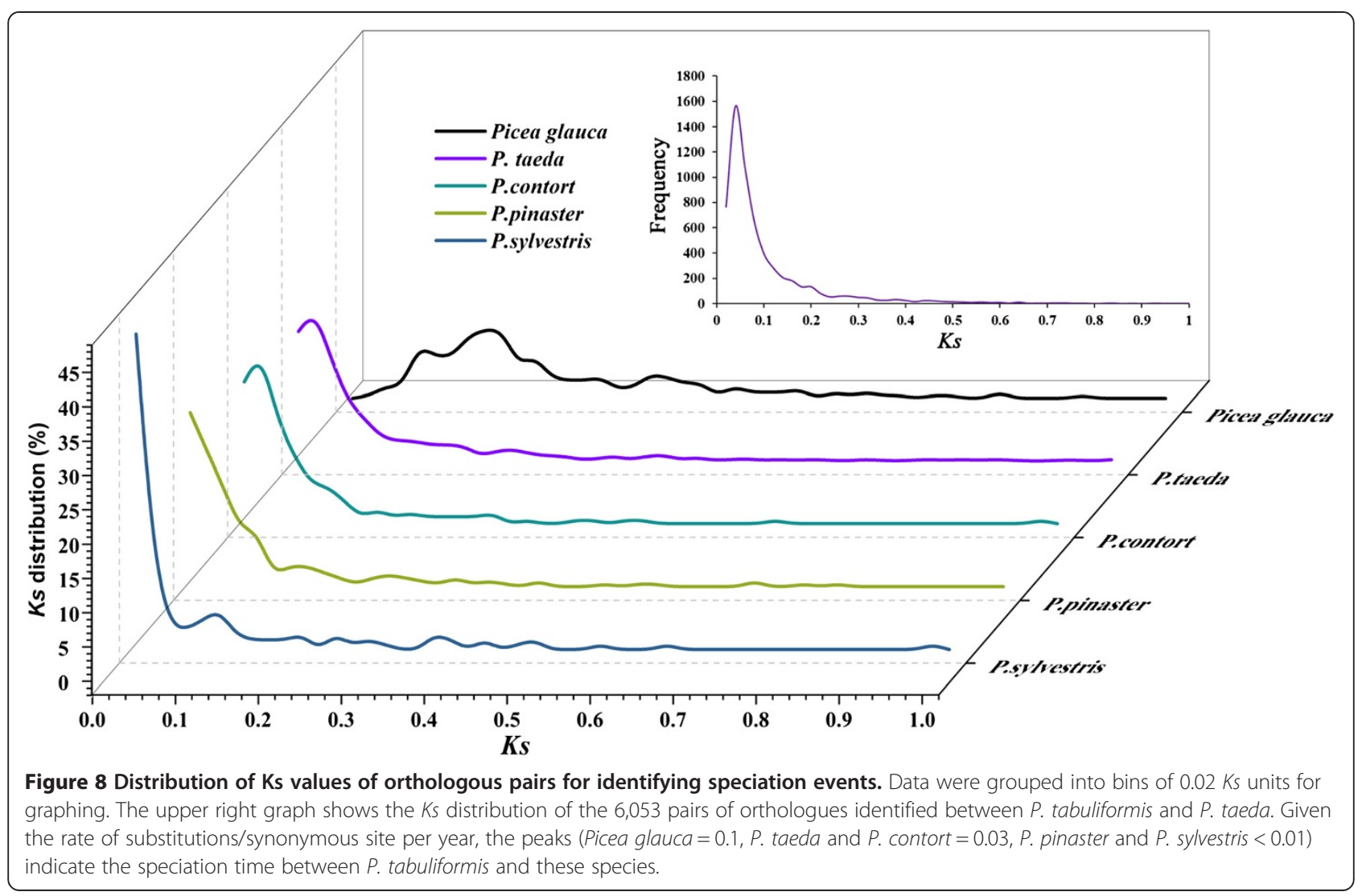




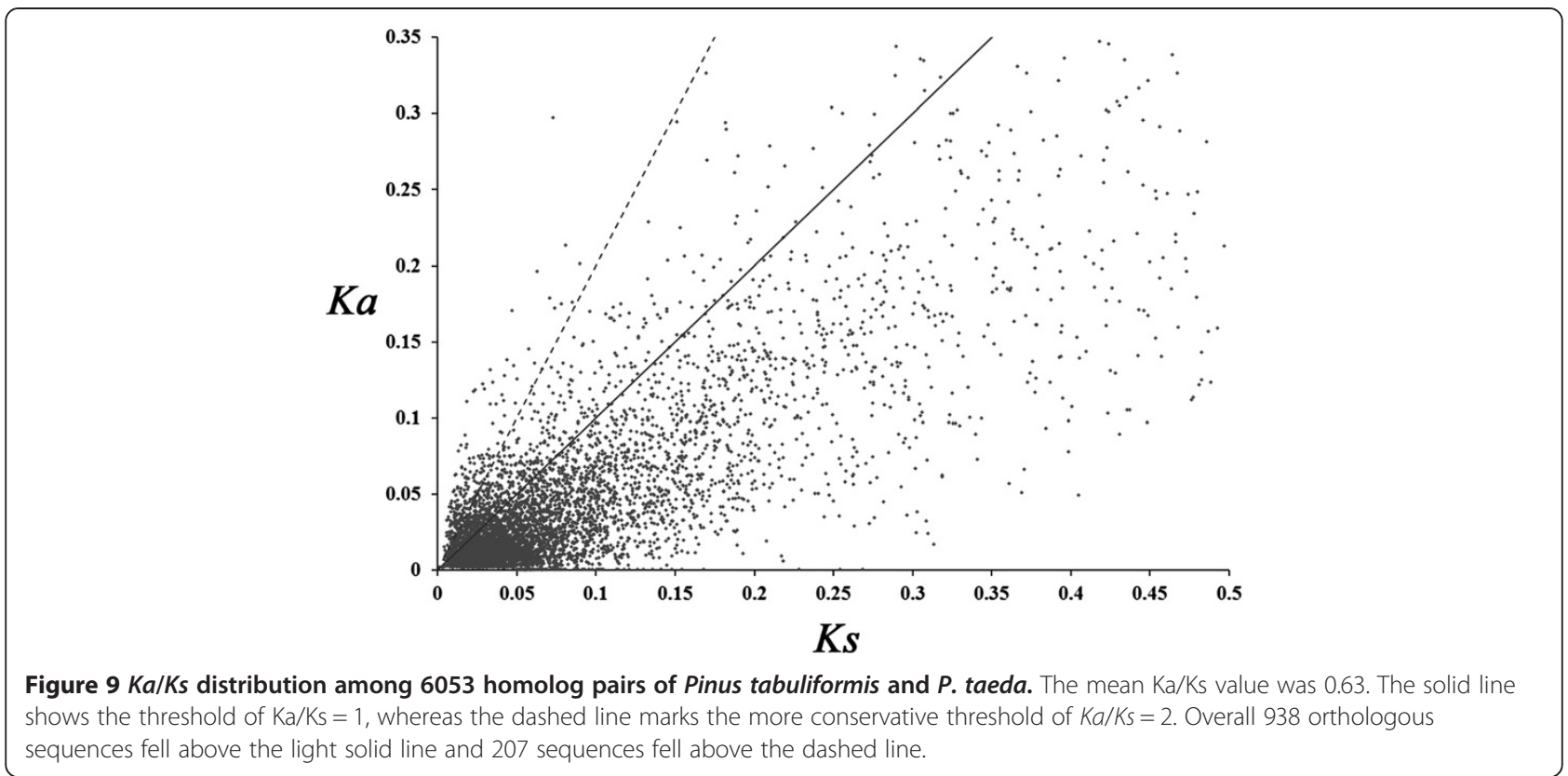

one normalised cDNA library yielded 822,891 ESTs in P. tabuliformis (Table 1). Although traditional sequencing yields longer EST sequences, it has little advantage compared to new assembly technology based on the large-scale ESTs. Additionally, most previous studies used a cDNA library of one tissue (Table 2), whereas we used a normalised cDNA library comprising multiple tissues and individuals. These large-scale ESTs will provide more comprehensive pine transcriptome information and facilitate the assembly of Pinus spp. ESTs in the future.

Next-generation sequencing technology yields a large number of sequences at considerably lower costs compared to traditional sequencing methods, and, therefore, provides a valuable starting point to expedite analysis of less-studied species [18,36,37]. Normalised cDNA libraries were used to sample large numbers of transcripts to maximise sequence diversity. Next-generation sequencing of normalised libraries is more efficient than that of nonnormalised libraries, particularly for rare transcripts [38].
The capacity to deliver large numbers of gene-based markers from transcriptome sequencing projects is a major advantage of next-generation sequencing technology $[18,20,36]$. Because of cost and throughput, conventional markers such as restriction fragment length polymorphism and random amplified polymorphic DNA are being replaced with SSRs and SNPs [20]. The genome-wide and abundant EST-based SSRs and SNPs/Indels markers obtained by next-generation sequencing represent an effective approach to marker discovery in many plant species, as these markers facilitate generation of dense genetic maps and have the advantage of higher cross-species transferability [6,39-41]. However, relevant studies in Pinus spp. are limited. In this study, 724 distinct EST-SSR loci and more than 92,000 SNPs/InDels were identified. It is possible to use these markers in a broad range of applications, including genetic mapping, genotype identification, marker-assisted selection breeding, and molecular tagging of genes. Among the EST-derived SSRs, tri-nucleotide repeat units were predominant. Considering the importance

Table 2 Transcriptome sequencing in Pinus spp.

\begin{tabular}{|c|c|c|c|c|c|c|}
\hline Pinus spp. & Platform or approach & Libraries & Reads or ESTs & Mean length & Unigenes & References \\
\hline P. taeda & cDNA clones & xylem & 1097 & 510 & 736 & {$[32]$} \\
\hline P. taeda & cDNA clones & xylem & 59797 & 364 & 20377 & [33] \\
\hline P. taeda & cDNA clones & root & 12918 & 555 & 6202 & {$[35]$} \\
\hline P. taeda & cDNA clones & embryos & 68721 & 689 & 12154 & [3] \\
\hline P. radiata & cDNA clones & xylem & 6389 & 624 & 3304 & [29] \\
\hline P. contorta & GS XL R70 & needles and conelets & 586732 & 306 & 17000 & [9] \\
\hline P. pinaster & Sanger and GS-FLX & different tissues & 951641 & 597 & 55332 & [2] \\
\hline P.densata & Illumina & needles & 3968794 & & 84950 & {$[67]$} \\
\hline
\end{tabular}


of maintaining reading frames to generating a polypeptide within a partially or fully active, it is no surprise that this observation is common for tri-nucleotide expansions (or their multiples) within translated regions [6,42,43]. As usual, comparisons of $P$. tabuliformis transcriptome SNPs show an excess of transitional over transversional substitutions. Similarly, A and $\mathrm{T}$ were the most frequent insertion and deletion types of Indels. Part of this bias is due to the relatively high rate of mutation of methylated cytosines to thymines $[44,45]$.

Comparative phylogenetic analysis at the genome level dramatically improves the precision and sensitivity of evolutionary inference [46]. However, comparative genomics in plants has been limited by the considerable phylogenetic distances between sequenced organisms [47]. Transcriptome sequencing using massively parallel sequencing technologies provides an attractive approach to obtaining large-scale sequence data for non-model organisms necessary for comparative genomic analysis $[24,48]$. Phylogenetic utility of transcriptome sequence data yields well-resolved and highly supported tree topologies for many groups of animals [49-51]; however, few such studies have been conducted with plant taxa [27]. Phylogenetic analysis of the genus Pinus has been limited mostly to plastid genome (cpDNA and mtDNA) sequences [21-23]. The results of this study are consistent with previous data on plastid genome phylogeny $[21,22]$, but transcriptome analyses, producing more robust results, are presented for the first time. Given that this study was not limited to particular genes or motifs, the results presented here are more representative of Pinus evolution than previous studies.

Understanding the factors that affect the evolutionary patterns and rates of genes is central in many research fields [52]. For the past 30 years, it was thought that the rate of gene evolution was determined by protein function [53]. Studies on yeast and bacteria indicate that the expression level of a protein affects the evolution rate more than its functional category, at least in unicellular species $[54,55]$. In this study, we have shown that sequence polymorphisms of the 191 putatively orthologous sets of ESTs of six Pinus species are widespread using GO terms. This suggests that selection of protein function does not contribute to the variation in the rates of gene evolution. However, most of the important factors are correlated with each other. More systematic analyses of genomic data are required to further demonstrate the effect of a range of factors on the evolutionary patterns and rates of genes.

\section{Conclusions}

This study is the first comprehensive sequencing effort and analysis of gene function in the transcriptome of $P$. tabuliformis and represents the most extensive expressed sequence resource available for $P$. tabuliformis to date. GO and KEGG analyses were carried out, and all unigenes were classified into functional categories so as to understand their functions and regulation pathways. An enormous number of SSR and SNP/Indel loci were detected. These data can be used to develop oligonucleotide microarrays or serve as a reference transcriptome for future RNA-seq experiments in large-scale gene expression assays. These data will accelerate our understanding of genetic variation in populations and the genetic control of important traits in P. tabuliformis. Additionally, the generation of such large-scale sequence data is a potentially invaluable scientific resource for mapping, marker-assisted breeding and conservationgenetic-oriented studies in P. tabuliformis and comparative evolutionary analysis of Pinus plants.

\section{Methods}

\section{Sample collection, cDNA library creation and 454 sequencing}

P. tabuliformis tissues were collected from 4-20 individual trees selected at random (genetically distinct) in a primary clonal Chinese pine seed orchard located in Xingcheng City, Liaoning Province, China (4044'N, 120³4'E, $100 \mathrm{~m}$ above sea level) [4]. The sampling time and number of individuals of each tissue type are listed in Table 3. Developing xylem tissues were scraped from the exposed xylem surface at breast height $(1.5 \mathrm{~m})$ after removing the bark from the sampling area. Samples were immediately placed in liquid nitrogen in the field until storage at $-80^{\circ} \mathrm{C}$.

Total RNA isolation from samples of all selected plant tissues, and cDNA library construction and normalisation were performed as described previously [56]. The pooled library was sequenced in a full 454 plate run on the GS-FLX Titanium platform (Roche, Indianapolis, IN, USA).

Table 3 Samples used for sequencing

\begin{tabular}{llccc}
\hline \multicolumn{1}{c}{ Samples in May } & Number of individuals & Samples in July & Number of individuals \\
\hline Tissue type & Cones & 10 & Strobili \\
\cline { 2 - 4 } & Cambium (stress side) & 4 & Cambium (stress side) \\
\cline { 2 - 4 } & Cambium (tension side) & 4 & Cambium (tension side) \\
\cline { 2 - 4 } & Cambium (stem) & 4 & Cambium (stem) \\
\cline { 2 - 4 } & Needles (juvenile + mature) & 20 & \\
\hline
\end{tabular}




\section{Assembly and annotation}

All generated ESTs were pre-screened to remove adaptor-ligated regions and contaminants by Seqclean and to trim low-quality regions by LUCY2 [57]. Because no reference $P$. tabuliformis genome exists, cleaned and qualified reads were assembled de novo in Newbler 2.5.3, which performs best for restoring full-length transcripts $[13,58]$.

The assembled isotig and singleton sequences were combined and clustered with CD-HIT (version 4.0) $[59,60]$. The sequences with similarity $>95 \%$ were divided into one class, and the longest sequence of each class was treated as a unigene during later processing. Descriptive annotations and GO classifications were performed as described previously [56].

We simultaneously instituted a search for putative unigenes against the NCBI protein database using a BLASTx and annotated each sequence with GO terms using Blast2GO.

\section{Identification of SSRs, SNPs and InDels}

Assembled isotigs with coverage of at least four reads were screened for SSRs, SNPs and InDels using Misa and ssahaSNP software, respectively [61]. Similar criteria for screening high-quality SNPs have been used in previous studies $[20,62]$. Only perfect repeats of two to six nucleotide repeats were identified. The minimum repeat-unit size for di-nucleotides was set at six and at five for tri- to hexa-nucleotide repeats.

\section{Identification of orthologues between six conifer species}

The shared transcriptome data of five conifer species in the PlantGDB and NCBI databases were downloaded. The numbers of unigenes for each species were as follows: Picea glauca $(48,619)$, P. contorta $(13,570)$, P. pinaster $(15,648)$, P. sylvestris $(73,609)$ and $P$. taeda (77,540). Along with 46,584 unigenes of P. tabuliformis, clustering was carried out among the transcribed sequences using UCLUST software [63]. Aligned sequences (at least $100 \mathrm{bp}$ ) showing 90\% identity were defined as pairs of putative orthologues among six species. The best-hit sequence of each cluster was then used in subsequent analyses. Orthologues of P. taeda and $P$. tabuliformis were searched using the same approach. Sequences of $P$. tabuliformis were annotated with GO terms using Blast2GO.

\section{Estimation at the level of synonymous substitution and} non-synonymous substitution between orthologues

Because unigenes are derived from EST sequences, have no annotated open reading frames and may contain frame shift sequencing errors, each member of a pair of sequences was searched using BLASTX against all plant protein sequences available in GenBank. The approach used was as described previously [64]. PAML software was used to estimate the non-synonymous substitutions per non-synonymous site $(\mathrm{Ka})$ and the synonymous substitutions per synonymous site $(K s)[65]$.

\section{Phylogenetic analysis}

Because the genus Pinus has a rich history of phylogenetic analysis and the relationships among the species in the genus are well understood [21-23], the precise topology is not critical for the purposes of this study. We chose to focus our analyses on the evolutionary pattern and rate of genes. The synonymous substitution and non-synonymous substitution between the orthologues of six conifer species were analysed as described previously. Phylograms were derived using pairwise substitution rates of orthologous transcripts as a distance metric with the neighbour-joining method [66]. Picea glauca was used as an out-group to root trees.

\section{Data availability}

The raw 454 EST data obtained in this study were deposited in the NCBI Sequence Read Archive (SRA) under the accession number SRA 056887.

\section{Additional files}

Additional file 1: The 191 orthologues of six conifer species.

Additional file 2: The 207 sequences with Ka/Ks ratios $>2$ of Pinus tabuliformis and $P$. taeda.

\section{Competing interests}

The authors declare that they have no competing interests.

\section{Authors' contributions}

SHN participated in the sequence alignment and drafted the manuscript. ZXL and HWY participated in the samples preparation and 454 sequencing. $X Y C$ and $Y L$ participated in the design of the study and performed the statistical analysis. WL conceived of the study, and participated in its design and coordination and helped to draft the manuscript. All authors read and approved the final manuscript.

\section{Acknowledgements}

This work was supported by grants from The Fundamental Research Funds for the Central Universities and the Special Fund for Scientific Forestry Research in the Public Interest (No.201104022).

\section{Author details}

${ }^{1}$ National Engineering Laboratory for Forest Tree Breeding, Key Laboratory for Genetics and Breeding of Forest Trees and Ornamental Plants of the Ministry of Education, College of Biological Science and Technology, Beijing Forestry University, Beijing 100083, People's Republic of China. ${ }^{2}$ Laboratory of Bio-technology of Tropical and Subtropical Forestry, College of Forestry, South China Agriculture University, Guangzhou 510642, People's Republic of China.

Received: 8 November 2012 Accepted: 15 April 2013 Published: 18 April 2013 


\section{References}

1. Ahuja MR, Neale DB: Evolution of genome size in conifers. Silvae Genet 2005, 54(3):126-137.

2. Fernandez-Pozo N, Canales J, Guerrero-Fernandez D, Villalobos DP, Diaz-Moreno SM, Bautista R, Flores-Monterroso A, Guevara MA, Perdiguero P, Collada C, et al: EuroPineDB: a high-coverage web database for maritime pine transcriptome. BMC Genomics 2011, 12:366.

3. Cairney J, Zheng L, Cowels A, Hsiao J, Zismann V, Liu J, Ouyang S, ThibaudNissen F, Hamilton J, Childs K, et al: Expressed sequence tags from loblolly pine embryos reveal similarities with angiosperm embryogenesis. Plant Mol Biol 2006, 62(4-5):485-501.

4. Li W, Wang X, Li Y: Stability in and correlation between factors influencing genetic quality of seed lots in seed orchard of Pinus tabuliformis Carr. over a 12-year span. PLoS One 2011, 6(8):e23544.

5. Chen K, Abbott RJ, Milne RI, Tian XM, Liu J: Phylogeography of Pinus tabulaeformis Carr. (Pinaceae), a dominant species of coniferous forest in northern China. Mol Ecol 2008, 17(19):4276-4288.

6. Lesser MR, Parchman TL, Buerkle CA: Cross-species transferability of SSR loci developed from transciptome sequencing in lodgepole pine. Mol Ecol Resour 2012, 12(3):448-455.

7. Ralph SG, Yueh H, Friedmann M, Aeschliman D, Zeznik JA, Nelson CC, Butterfield YS, Kirkpatrick R, Liu J, Jones SJ, et al: Conifer defence against insects: microarray gene expression profiling of Sitka spruce (Picea sitchensis) induced by mechanical wounding or feeding by spruce budworms (Choristoneura occidentalis) or white pine weevils (Pissodes strobi) reveals large-scale changes of the host transcriptome. Plant Cell Environ 2006, 29(8):1545-1570.

8. Valledor L, Jorrin JV, Rodriguez JL, Lenz C, Meijon M, Rodriguez R, Canal MJ: Combined proteomic and transcriptomic analysis identifies differentially expressed pathways associated to Pinus radiata needle maturation. J Proteome Res 2010, 9(8):3954-3979.

9. Parchman TL, Geist KS, Grahnen JA, Benkman CW, Buerkle CA: Transcriptome sequencing in an ecologically important tree species: assembly, annotation, and marker discovery. BMC Genomics 2010, 11:180.

10. Morse AM, Peterson DG, Islam-Faridi MN, Smith KE, Magbanua Z, Garcia SA, Kubisiak TL, Amerson HV, Carlson JE, Nelson CD, et al: Evolution of genome size and complexity in Pinus. PLoS One 2009, 4(2):e4332.

11. Neale DB: Genomics to tree breeding and forest health. Curr Opin Genet Dev 2007, 17(6):539-544.

12. Emrich SJ, Barbazuk WB, Li L, Schnable PS: Gene discovery and annotation using LCM-454 transcriptome sequencing. Genome Res 2007, 17(1):69-73.

13. Mundry M, Bornberg-Bauer E, Sammeth M, Feulner PG: Evaluating characteristics of de novo assembly software on 454 transcriptome data: a simulation approach. PLoS One 2012, 7(2):e31410.

14. Hiremath PJ, Farmer A, Cannon SB, Woodward J, Kudapa H, Tuteja R, Kumar A, Bhanuprakash A, Mulaosmanovic B, Gujaria N, et al: Large-scale transcriptome analysis in chickpea (Cicer arietinum L.), an orphan legume crop of the semi-arid tropics of Asia and Africa. Plant Biotechnol J 2011, 9(8):922-931.

15. Fraser BA, Weadick $C J$, Janowitz I, Rodd FH, Hughes KA: Sequencing and characterization of the guppy (Poecilia reticulata) transcriptome. BMC Genomics 2011, 12:202.

16. Russell JR, Bayer M, Booth C, Cardle L, Hackett CA, Hedley PE, Jorgensen L, Morris JA, Brennan RM: Identification, utilisation and mapping of novel transcriptome-based markers from blackcurrant (Ribes nigrum). BMC Plant Biol 2011, 11:147.

17. Wang Y, Zeng X, lyer NJ, Bryant DW, Mockler TC, Mahalingam R: Exploring the switchgrass transcriptome using second-generation sequencing technology. PLoS One 2012, 7(3):e34225.

18. Kaur S, Pembleton LW, Cogan NO, Savin KW, Leonforte T, Paull J, Materne M, Forster JW: Transcriptome sequencing of field pea and faba bean for discovery and validation of SSR genetic markers. BMC Genomics 2012, 13:104

19. Renaut S, Nolte AW, Bernatchez L: Mining transcriptome sequences towards identifying adaptive single nucleotide polymorphisms in Lake Whitefish species pairs (Coregonus spp. Salmonidae). Mol Ecol 2010, 19(Suppl 1):115-131.

20. Barbazuk WB, Emrich SJ, Chen HD, Li L, Schnable PS: SNP discovery via 454 transcriptome sequencing. Plant J 2007, 51(5):910-918

21. Eckert AJ, Hall BD: Phylogeny, historical biogeography, and patterns of diversification for Pinus (Pinaceae): phylogenetic tests of fossil-based hypotheses. Mol Phylogenet Evol 2006, 40(1):166-182.
22. Gernandt DS, Lopez GG, Garcia SO, Liston A: Phylogeny and classification of Pinus. Taxon 2005, 54(1):29-42.

23. Wang B, Mao JF, Gao J, Zhao W, Wang XR: Colonization of the Tibetan Plateau by the homoploid hybrid pine Pinus densata. Mol Ecol 2011 20(18):3796-3811.

24. Der JP, Barker MS, Wickett NJ, DePamphilis CW, Wolf PG: De novo characterization of the gametophyte transcriptome in bracken fern, Pteridium aquilinum. BMC Genomics 2011, 12:99.

25. DiGuistini S, Ralph SG, Lim YW, Holt R, Jones S, Bohlmann J, Breuil C: Generation and annotation of lodgepole pine and oleoresin-induced expressed sequences from the blue-stain fungus Ophiostoma clavigerum, a Mountain Pine Beetle-associated pathogen. FEMS Microbiol Lett 2007, 267(2):151-158.

26. Heller G, Adomas A, Li G, Osborne J, van Zyl L, Sederoff R, Finlay RD, Stenlid J, Asiegbu FO: Transcriptional analysis of Pinus sylvestris roots challenged with the ectomycorrhizal fungus Laccaria bicolor. BMC Plant Biol 2008, 8:19.

27. Logacheva MD, Kasianov AS, Vinogradov DV, Samigullin TH, Gelfand MS, Makeev VJ, Penin AA: De novo sequencing and characterization of floral transcriptome in two species of buckwheat (Fagopyrum). BMC Genomics 2011, 12:30

28. Buschiazzo E, Ritland C, Bohlmann J, Ritland K: Slow but not low: genomic comparisons reveals slower evolutionary rate and higher $d N / d S$ in conifers compared to angiosperms. BMC Evol Biol 2012, 12(1):8.

29. Li X, Wu HX, Dillon SK, Southerton SG: Generation and analysis of expressed sequence tags from six developing xylem libraries in Pinus radiata D. Don. BMC Genomics 2009, 10:41.

30. Li X, Wu HX, Southerton SG: Transcriptome profiling of Pinus radiata juvenile wood with contrasting stiffness identifies putative candidate genes involved in microfibril orientation and cell wall mechanics. BMC Genomics 2011, 12:480

31. Li X, Wu HX, Southerton SG: Seasonal reorganization of the xylem transcriptome at different tree ages reveals novel insights into wood formation in Pinus radiata. New Phytol 2010, 187(3):764-776.

32. Allona I, Quinn M, Shoop E, Swope K, St CS, Carlis J, Riedl J, Retzel E, Campbell MM, Sederoff $R$, et al: Analysis of xylem formation in pine by cDNA sequencing. Proc Natl Acad Sci U S A 1998, 95(16):9693-9698.

33. Kirst M, Johnson AF, Baucom C, Ulrich E, Hubbard K, Staggs R, Paule C, Retzel E, Whetten R, Sederoff R: Apparent homology of expressed genes from wood-forming tissues of loblolly pine (Pinus taeda L.) with Arabidopsis thaliana. Proc Natl Acad Sci U S A 2003, 100(12):7383-7388.

34. Pavy N, Laroche J, Bousquet J, Mackay J: Large-scale statistical analysis of secondary xylem ESTs in pine. Plant Mol Biol 2005, 57(2):203-224.

35. Lorenz WW, Sun F, Liang C, Kolychev D, Wang H, Zhao X, Cordonnier-Pratt MM, Pratt LH, Dean JF: Water stress-responsive genes in loblolly pine (Pinus taeda) roots identified by analyses of expressed sequence tag libraries. Tree Physiol 2006, 26(1):1-16.

36. Jhanwar S, Priya P, Garg R, Parida SK, Tyagi AK, Jain M: Transcriptome sequencing of wild chickpea as a rich resource for marker development. Plant Biotechnol J 2012, 10(6):690-702.

37. Edwards CE, Parchman TL, Weekley CW: Assembly, gene annotation and marker development using 454 floral transcriptome sequences in Ziziphus celata (Rhamnaceae), a highly endangered, Florida endemic plant. DNA Res 2012, 19(1):1-9.

38. Wall PK, Leebens-Mack J, Chanderbali AS, Barakat A, Wolcott E, Liang H, Landherr $L$, Tomsho $L P$, Hu Y, Carlson JE, et al: Comparison of next generation sequencing technologies for transcriptome characterization. BMC Genomics 2009, 10:347.

39. Ellis JR, Burke JM: EST-SSRs as a resource for population genetic analyses. Heredity (Edinb) 2007, 99(2):125-132.

40. Barbara T, Palma-Silva C, Paggi GM, Bered F, Fay MF, Lexer C: Cross-species transfer of nuclear microsatellite markers: potential and limitations. Mol Ecol 2007, 16(18):3759-3767.

41. Bouck A, Vision T: The molecular ecologist's guide to expressed sequence tags. Mol Ecol 2007, 16(5):907-924.

42. Rowland LJ, Alkharouf N, Darwish O, Ogden EL, Polashock JJ, Bassil NV, Main D: Generation and analysis of blueberry transcriptome sequences from leaves, developing fruit, and flower buds from cold acclimation through deacclimation. BMC Plant Biol 2012, 12(1):46.

43. Blanca J, Canizares J, Roig C, Ziarsolo P, Nuez F, Pico B: Transcriptome characterization and high throughput SSRs and SNPs discovery in Cucurbita pepo (Cucurbitaceae). BMC Genomics 2011, 12:104. 
44. Zhao Z, Boerwinkle E: Neighboring-nucleotide effects on single nucleotide polymorphisms: a study of 2.6 million polymorphisms across the human genome. Genome Res 2002, 12(11):1679-1686.

45. Keller I, Bensasson D, Nichols RA: Transition-transversion bias is not universal: a counter example from grasshopper pseudogenes. PLoS Genet 2007, 3(2):e22

46. Clark AG, Eisen MB, Smith DR, Bergman CM, Oliver B, Markow TA, Kaufman TC, Kellis M, Gelbart W, lyer VN, et al: Evolution of genes and genomes on the Drosophila phylogeny. Nature 2007, 450(7167):203-218.

47. Kunstner A, Wolf JB, Backstrom N, Whitney O, Balakrishnan CN, Day L, Edwards SV, Janes DE, Schlinger BA, Wilson RK, et al: Comparative genomics based on massive parallel transcriptome sequencing reveals patterns of substitution and selection across 10 bird species. Mol Ecol 2010, 19(Suppl 1):266-276.

48. Zakas C, Schult N, McHugh D, Jones KL, Wares JP: Transcriptome analysis and SNP development can resolve population differentiation of Streblospio benedicti, a developmentally dimorphic marine annelid. PLOS One 2012, 7(2):e31613.

49. Meusemann K, von Reumont BM, Simon S, Roeding F, Strauss S, Kuck P, Ebersberger I, Walzl M, Pass G, Breuers S, et al: A phylogenomic approach to resolve the arthropod tree of life. Mol Biol Evol 2010, 27(11):2451-2464.

50. Roeding F, Borner J, Kube M, Klages S, Reinhardt R, Burmester T: A 454 sequencing approach for large scale phylogenomic analysis of the common emperor scorpion (Pandinus imperator). Mol Phylogenet Evol 2009, 53(3):826-834.

51. Roeding F, Hagner-Holler S, Ruhberg H, Ebersberger I, von Haeseler A, Kube M, Reinhardt R, Burmester T: EST sequencing of Onychophora and phylogenomic analysis of Metazoa. Mol Phylogenet Evol 2007, 45(3):942-951.

52. Pal C, Papp B, Lercher MJ: An integrated view of protein evolution. Nat Rev Genet 2006, 7(5):337-348

53. Mclnerney JO: The causes of protein evolutionary rate variation. Trends Ecol Evol 2006, 21(5):230-232

54. Rocha EP: The quest for the universals of protein evolution. Trends Genet 2006, 22(8):412-416.

55. Wall DP, Hirsh AE, Fraser HB, Kumm J, Giaever G, Eisen MB, Feldman MW: Functional genomic analysis of the rates of protein evolution. Proc Nat Acad Sci U S A 2005, 102(15):5483-5488.

56. Garzon-Martinez GA, Zhu I, Landsman D, Barrero LS, Marino-Ramirez L: The Physalis peruviana leaf transcriptome: assembly, annotation and gene model prediction. BMC Genomics 2012, 13(1):151.

57. Li S, Chou HH: LUCY2: an interactive DNA sequence quality trimming and vector removal tool. Bioinformatics 2004, 20(16):2865-2866

58. Kumar S, Blaxter ML: Comparing de novo assemblers for 454 transcriptome data. BMC Genomics 2010, 11:571.

59. Huang Y, Niu B, Gao Y, Fu L, Li W: CD-HIT Suite: a web server for clustering and comparing biological sequences. Bioinformatics 2010, 26(5):680-682.

60. Li W, Godzik A: Cd-hit: a fast program for clustering and comparing large sets of protein or nucleotide sequences. Bioinformatics 2006, 22(13):1658-1659.

61. van Oeveren J, Janssen A: Mining SNPs from DNA sequence data; computational approaches to SNP discovery and analysis. Methods $\mathrm{Mol}$ Biol 2009, 578:73-91

62. Milano I, Babbucci M, Panitz F, Ogden R, Nielsen RO, Taylor MI, Helyar SJ, Carvalho GR, Espineira M, Atanassova M, et al: Novel tools for conservation genomics: comparing two high-throughput approaches for SNP discovery in the transcriptome of the European hake. PLoS One 2011, 6(11):e28008.

63. Edgar RC: Search and clustering orders of magnitude faster than BLAST. Bioinformatics 2010, 26(19):2460-2461.

64. Blanc G, Wolfe KH: Widespread paleopolyploidy in model plant species inferred from age distributions of duplicate genes. Plant Cell 2004, 16(7):1667-1678

65. Yang Z: PAML 4: phylogenetic analysis by maximum likelihood. $\mathrm{Mol}$ Biol Evol 2007, 24(8):1586-1591.
66. Tamura K, Dudley J, Nei M, Kumar S: MEGA4: molecular evolutionary genetics analysis (MEGA) software version 4.0. Mol Biol Evol 2007, 24(8):1596-1599.

67. Wan LC, Zhang H, Lu S, Zhang L, Qiu Z, Zhao Y, Zeng QY, Lin J: Transcriptome-wide identification and characterization of miRNAs from Pinus densata. BMC Genomics 2012, 13:132.

doi:10.1186/1471-2164-14-263

Cite this article as: Niu et al:: Transcriptome characterisation of Pinus tabuliformis and evolution of genes in the Pinus phylogeny. BMC Genomics 2013 14:263.

\section{Submit your next manuscript to BioMed Central and take full advantage of:}

- Convenient online submission

- Thorough peer review

- No space constraints or color figure charges

- Immediate publication on acceptance

- Inclusion in PubMed, CAS, Scopus and Google Scholar

- Research which is freely available for redistribution

Submit your manuscript at www.biomedcentral.com/submit
C Biomed Central 